\title{
National Hospital Care Survey Demonstration Projects: Examination of Inpatient Hospitalization and Risk of Mortality Among Patients Diagnosed With Pneumonia
}

\author{
Zachary J. Peters, M.P.H., Jill J. Ashman, Ph.D., Alexander Schwartzman, and Carol J. DeFrances, Ph.D.
}

\section{Abstract}

Objective-This report demonstrates the ability of data from the National Hospital Care Survey (NHCS) linked to the National Death Index (NDI) to provide information on inpatient hospitalizations and in-hospital and post-acute mortality among patients hospitalized for a specific condition, in this case pneumonia.

Methods-The 2016 NHCS consists of information on all hospitalizations provided by participating hospitals. NHCS collects personally identifiable information, allowing for linkage across hospital settings and to outside data sources, including NDI; 97 hospitals in the 2016 NHCS contributed qualifying patient records that were eligible to be linked to NDI. Exploratory analyses were conducted to assess mortality outcomes, describe cause of death, and model the risk of in-hospital and post-acute mortality among hospitalized patients diagnosed with pneumonia. NHCS data are unweighted and are not nationally representative.

Results - Nearly 77,000 adult patients were hospitalized for pneumonia in the 2016 NHCS and were eligible for linkage to NDI. Overall, $35 \%$ of patients hospitalized with pneumonia died either in the hospital or within 1 year of discharge. Pneumonia patients who were admitted to the intensive care unit (ICU) and eventually discharged had a $41 \%$ increased risk of death within 30 days of discharge than those not admitted to ICU, after adjusting for sex, age, and length of stay (adjusted risk ratio is $1.41,95 \%$ confidence interval is $1.35-1.48$ ).

Conclusion - Although the findings are not nationally representative, this report demonstrates the potential of NHCS data linked to NDI to identify specific patient cohorts, assess mortality outcomes, and estimate the risk of in-hospital and post-acute mortality.

Keywords: inpatients $\bullet$ pneumonia $\bullet$ mortality risk $\bullet$ National Death Index

\section{Introduction}

Disease-specific mortality has decreased among hospitalized patients over the last 2 decades $(1,2)$, but information on posthospitalization, or post-acute, mortality among patients is limited as most national hospitalization data sets do not capture subsequent hospitalizations, emergency department visits, or mortality outcomes.

To address these limitations, the National Center for Health Statistics (NCHS) launched the National Hospital
Care Survey (NHCS), which is designed to collect inpatient and ambulatory encounter data from a large sample of hospitals in the United States. Through NCHS' data linkage program and funding from the Patient-Centered Outcomes Research Trust Fund, NCHS has expanded the analytic use of NHCS by linking it to the National Death Index (NDI), which provides information such as date of death and underlying and multiple causes of death among patients who died posthospital discharge. NHCS-participating hospitals provided data on all hospitalized patients and patients who made emergency department (ED) or outpatient department (OPD) visits for all of 2016. NHCS data contain the discharge status for hospitalized patients, allowing for the assessment of in-hospital mortality. However, with NHCS data linked to NDI, patients seeking care for a given condition in EDs or inpatient settings can be followed prospectively to assess subsequent mortality status (and for deceased patients, the timing and underlying and multiple causes of death). Few other data sets have the capability to assess subsequent post-acute mortality after a hospitalization. Linking health care utilization data with mortality data provides a platform to assess patients' risk for death, thereby providing

\section{U.S. DEPARTMENT OF HEALTH AND HUMAN SERVICES Centers for Disease Control and Prevention National Center for Health Statistics}


information to inform opportunities to intervene during care delivery, improve patient monitoring postdischarge, and consider policies to improve mortality outcomes.

This report demonstrates the ability of NHCS-NDI linked data to perform the following analyses: a) identify hospitalized patients according to a specific condition or disease, $b$ ) assess mortality outcomes among those patients out to 1 year postdischarge, c) evaluate causes of death among deceased patients, and d) model risk of death associated with various patient and hospitalization characteristics.

Specifically, this report assesses in-hospital and post-acute mortality for adult patients diagnosed with pneumonia. Pneumonia patients were included in this report as an example of a common cause of hospitalization; in 2018, pneumonia was the fourth most common principal diagnosis among inpatients, excluding births (3). Additionally, acute lower respiratory disease (pneumonia and influenza) routinely is one of the top 10 leading causes of death in the United States (4). Several cohort studies have assessed various aspects of mortality and mortality risk during and after hospitalizations for pneumonia in the United States (5-10), including a recent study of a large, national, electronic health record data set that assessed overall mortality and risk of mortality among adults in the year following hospitalization for pneumonia from 2012-2018 (11). All-cause mortality within 1 year of admission was $18 \%$ and increased with age and severity of comorbidities; however, cause-specific mortality was not assessed, and mortality status was ascertained from the initial and subsequent hospital admissions, likely missing out-of-hospital deaths. This study seeks to build on findings from these studies by using a large sample of hospitalizations linked to official mortality data to assess overall and cause-specific mortality, timing of death, and risk factors for death after pneumonia-related hospitalizations. Although NHCS data are unweighted and are not nationally representative, the purpose of this report is to demonstrate the analytical capabilities of NHCS and NDI linked data set.

\section{Methods}

\section{Data sources}

\section{National Hospital Care Survey}

The target universe for NHCS is inpatient discharges and in-person visits made to EDs and OPDs in noninstitutional and nonfederal hospitals in the 50 states and the District of Columbia that have six or more staffed inpatient beds. Data are extracted from hospital billing or electronic health record systems and then transmitted electronically directly to NCHS or its designated agent. The 2016 sample consisted of 581 hospitals: 506 were acute care hospitals and 75 were other specialty hospitals, including children's, psychiatric, long-term acute care, and rehabilitation hospitals. Participation in NHCS is voluntary. Since the launch of the survey in 2011, the participation rate has remained relatively low and was $27 \%$ in 2016, with 158 out of the initial 581 sample hospitals participating. Because of the low participation rate, the data are unweighted and are not nationally representative.

Of the 158 participating hospitals, $93 \%$ were acute care, with a small number of psychiatric, children's, long-term acute-care, and rehabilitation hospitals making up the remaining $7 \%$ of the sample. This report focused solely on data from inpatient stays in the 2016 NHCS. Participating hospitals were asked to provide data on all hospitalizations during the 2016 calendar year, representing an unweighted total of 2,591,722 hospitalizations. More information on NHCS methodology is available elsewhere (12). NHCS collects personally identifiable information (PII) (for example, patient name and date of birth), which allows for both following patients across hospital settings and linkage to external data sources.

\section{National Hospital Care Survey data linked to the 2016-2017 National Death Index}

Through its data linkage program, NCHS has expanded the analytic use of the data collected from NHCS by linking it with mortality data from NDI, a component of the National Vital Statistics System (13). NDI is a centralized database of death record information compiled from state vital statistics offices. In collaboration with the states, NCHS established NDI as a resource for epidemiological follow-up studies and other types of health and medical research that require determination of the mortality status of study subjects. These mortality data are provided by the states under contract agreements with NCHS that specify how these data may be used, for what purposes, and at what cost. Currently, NDI contains about 85 million records from 1979 through 2018 from the 50 states, the District of Columbia, Puerto Rico, and the U.S. Virgin Islands.

The linkage between 2016 NHCS patient records and 2016-2017 NDI death certificate records was based on both deterministic and probabilistic methods. The probabilistic linkage method performed weighting and link adjudication as described in the FellegiSunter paradigm, the foundational methodology used for record linkage (14). More information on NHCS-NDI linkage methodology and the process for determining patient mortality status is available elsewhere (15).

\section{Analytic sample}

This report includes patients aged 18 and over who were hospitalized with a pneumonia diagnosis in the 2016 NHCS. Overall, 158 hospitals participated in the 2016 NHCS, of which 145 submitted inpatient hospitalization records.

Hospitalizations were excluded from the analysis if age, sex, date of discharge, diagnosis code, or discharge code were missing (27\%). Seventeen hospitals did not submit any inpatient records that met inclusion criteria and accounted for $26 \%$ of all excluded hospitalizations. The remaining 128 hospitals contributed qualifying inpatient records with complete data in these fields, however, 121 of those hospitals also submitted nonqualifying records that did not meet inclusion criteria.

Additionally, only patients with sufficient PII for linkage with NDI were included. Linkage eligibility was defined as having usable information for two of the three data element groups: Social Security number, name, and date 
of birth (year, month, day) (15); 97 hospitals contributed inpatient records that met the definition of a qualifying hospitalization among patients who were linkage eligible. For linkage-eligible patients with more than one qualifying hospitalization for pneumonia in 2016, analyses were based on their most recent hospitalization. Two-thirds of patients with a qualifying pneumonia hospitalization (67\%) were eligible to be linked to NDI. Nearly all pneumonia patients who were ineligible for linkage were from hospitals that did not provide any patient records with PII (98\%). Among linkage-eligible patients who died in the hospital according to their discharge code, $100 \%$ matched to an NDI record indicating the patient died. Although the linked NHCS-NDI data set provides up to 2 years of mortality data, the current study restricted mortality assessment postdischarge to 1 year so that all patients alive after a 2016 hospitalization had an equal follow-up period in which death could occur.

\section{Inpatient stays for pneumonia}

Hospitalizations for pneumonia were identified as having an International Classification of Diseases, 10th Revision, Clinical Modification (ICD-10-CM) code between J12-J18 listed in any diagnosis variable. For some NHCS sampled hospitals it was not possible to identify the primary diagnosis, and for other hospitals there were no present on admission indicators. Therefore, all available diagnoses were used to identify patients hospitalized with pneumonia in this report. Among all pneumonia hospitalizations with these indicators, only $22 \%$ specified pneumonia as the primary diagnosis, while $87 \%$ indicated that the pneumonia diagnosis was present on admission.

\section{Mortality outcomes}

Mortality was assessed for all linkage-eligible patients with a qualifying hospitalization in 2016. Mortality outcomes include: in-hospital death; post-acute deaths occurring 0-30, 31-60, 61-90, and 91-365 days posthospital discharge; and alive at 366 days postdischarge. In-hospital death was assigned to patients with an NHCS discharge code signifying death. Among patients who were alive at discharge, post-acute mortality outcomes were assigned when patients linked to NDI and NDI death date minus the NHCS discharge date was less than or equal to 365 days. Patients were treated as alive after 1 year if their NHCS discharge status did not indicate in-hospital death and they did not link to a death record in the NDI data within 365 days of their hospital discharge.

\section{Cause of death}

Cause of death for in-hospital and post-acute mortality was assessed using the 113 Underlying Cause-of-Death Recodes Adapted for Use by NCHS' Division of Vital Statistics in NDI (16). Cause-of-death categories were included based on their prevalence among deceased patients in this cohort (for example, malignant neoplasms, cardiovascular disease, and chronic lower respiratory disease) or based on their relevance to this analysis (for example, pneumonia). A death with any mention of the following codes in either the contributing or underlying cause of death was categorized as follows:

- Cardiovascular disease recodes 053-069, 071-075

- Chronic lower respiratory disease recodes $082-086$

- Malignant neoplasm recodes 019-043

- Pneumonia recodes 076, 078

All cause-of-death codes not listed above were grouped into an "other" category, including other respiratory diseases $(5 \%$; includes influenza, pneumonitis due to solids and liquids, and pneumoconiosis), cerebrovascular disease $(4 \%)$, septicemia $(3 \%)$, renal failure (3\%), and diabetes mellitus (3\%). All other specific cause-of-death codes accounted for $2 \%$ or fewer of deaths in this cohort.

\section{Statistical analysis}

Mortality outcomes were assessed among linkage-eligible patients hospitalized for pneumonia, overall and by age cohort, including patients aged $18-44,45-64$, and 65 and over. Cause of death among deceased patients was assessed within each timing-of-death category.
The risk of in-hospital and post-acute mortality was then modeled using hospitalization and patient characteristics. Log-binomial regression was used to estimate the following associations:

- Risk of in-hospital death by intensive care unit (ICU) status (admitted to ICU during hospitalization compared with not admitted to ICU) among all patients hospitalized with pneumonia

- Risk of death within 1 year by ICU status among pneumonia patients who were discharged from the hospital.

Lastly, multivariate Poisson regression with robust error variance was used, as described by Zou (17) and Knol, et al. (18), to model the adjusted risk of death within 30 days among pneumonia patients who were discharged from the hospital. This model included independent variables of age, sex, length of inpatient stay, and ICU status. Age was treated as continuous, with patients over age 97 set equal to 97 years $(n=553,1 \%)$. Length of stay was treated as continuous with hospital stays over 60 days set equal to 60 days $(n=480,1 \%)$. Risk ratios (RRs), standard errors, and $95 \%$ confidence intervals (CIs) are shown.

SAS version 9.4 (SAS Institute, Cary, N.C.) was used for all data analyses. These analyses are descriptive only and are intended to represent examples of analyses that can be performed with these data. Any statistical comparisons were included solely to illustrate the capabilities of the data set, not to produce official, representative estimates for the condition examined. Statements about the relative magnitude of numbers or percentages in this report do not imply statistical significance.

\section{Results}

There were 130,657 qualifying pneumonia hospitalizations at an NHCS hospital in 2016 among 114,090 patients (data not shown). Of those, 89,448 qualifying hospitalizations were among patients who were eligible to be linked to NDI, resulting in a final cohort of 76,857 patients with a qualifying hospitalization who were linkage-eligible (Table 1). More than one-third of linkage-eligible patients with pneumonia (35\%) died 
during their hospitalization or within 1 year of their hospital discharge (Table 1).

\section{Mortality outcomes}

Figure 1 depicts mortality outcomes among patients with a linkage-eligible hospitalization for pneumonia.

- Among patients of all ages, 9\% died in the hospital, 9\% died 0-30 days after discharge, 16\% died 31-365 days after discharge, and 65\% were alive at 366 days postdischarge.

- The percentage of patients who were alive 1 year after discharge decreased with age, with $86 \%$ of patients aged $18-44,73 \%$ of patients aged $45-64$, and $57 \%$ of patients aged 65 and over alive at 366 days postdischarge.

- The percentage of patients who died during a hospitalization for pneumonia increased with age (5\% of patients aged $18-44,8 \%$ of patients $45-64$, and $11 \%$ of patients 65 and over).

\section{Cause of death}

Figure 2 presents the cause of death for pneumonia patients who died in the hospital or within 1 year of discharge.

- Pneumonia as a cause of death was more prevalent among patients who died in the hospital (11\%) compared with patients who died 0-30 days (3\%), 31-60 days (3\%), 61-90 days (3\%), and $91-365$ days $(2 \%)$ postdischarge.

- Cardiovascular disease as a cause of death increased from $16 \%$ of pneumonia patients who died in the hospital to $24 \%$ of pneumonia patients who died 90-365 days postdischarge.

- Similarly, chronic lower respiratory disease as a cause of death increased from $7 \%$ of pneumonia patients who died in the hospital to $11 \%$ of pneumonia patients who died 90-365 days postdischarge.

- For all deaths combined, malignant neoplasm was the most common underlying cause of death for pneumonia patients who died in the hospital or within 1 year of discharge $(26 \%)$. This was true for each timing-of-death category except 91-365 days, where malignant neoplasms and cardiovascular disease both accounted for $24 \%$ of deaths occurring at that time.

\section{Risk of death}

Table 2 shows the risk of in-hospital death by ICU status among all patients hospitalized for pneumonia.

- Pneumonia patients who were admitted to ICU had nearly four times the risk of dying in the hospital than patients who were not admitted to ICU (RR: 3.77, 95\% CI: 3.57-3.98).

Table 3 shows the mortality risk within 365 days after discharge by ICU status among pneumonia patients who were discharged alive.

\section{Figure 1. Mortality outcomes among hospitalized pneumonia adult patients, by age}

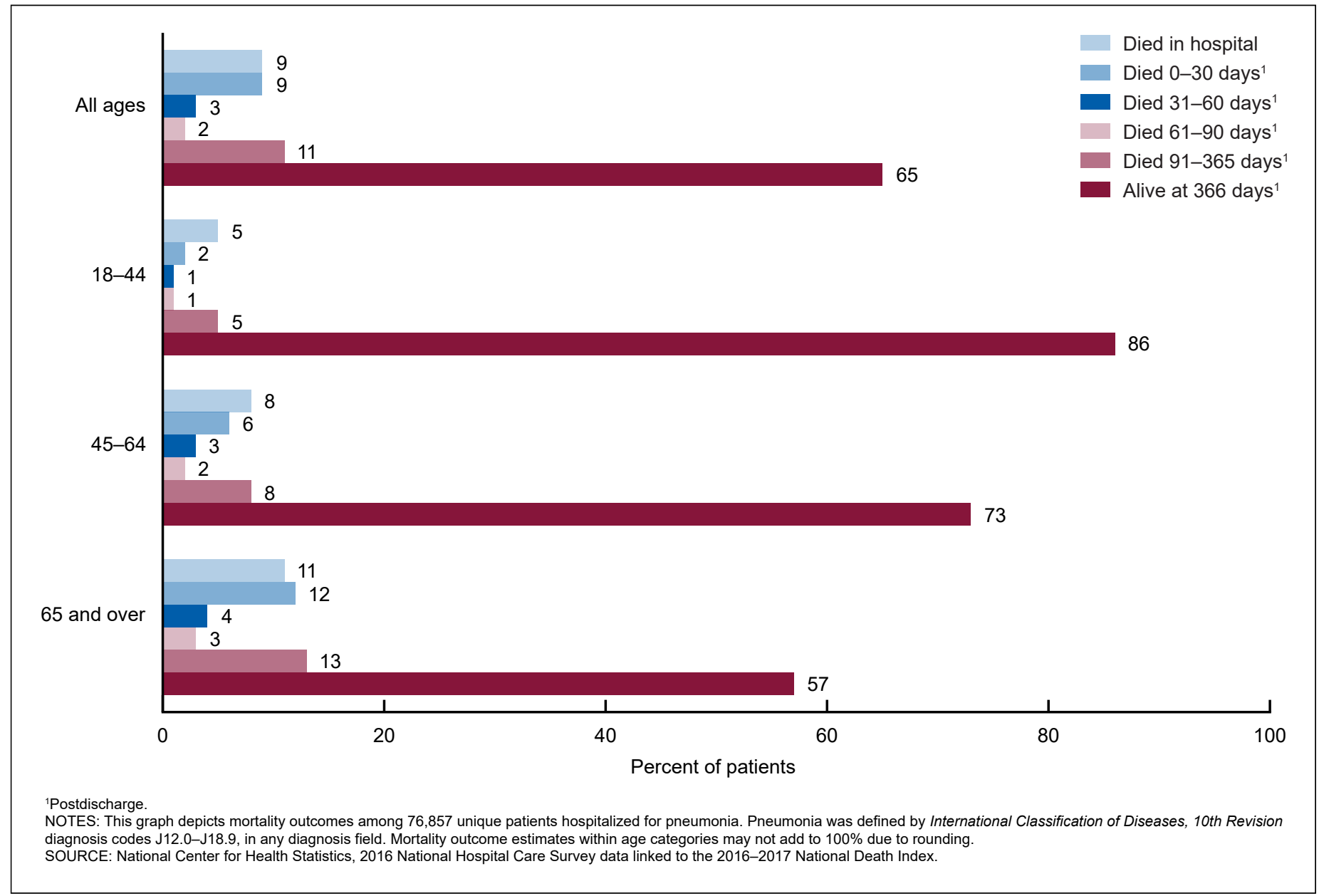


Figure 2. Causes of death among deceased pneumonia adult patients, by timing of death

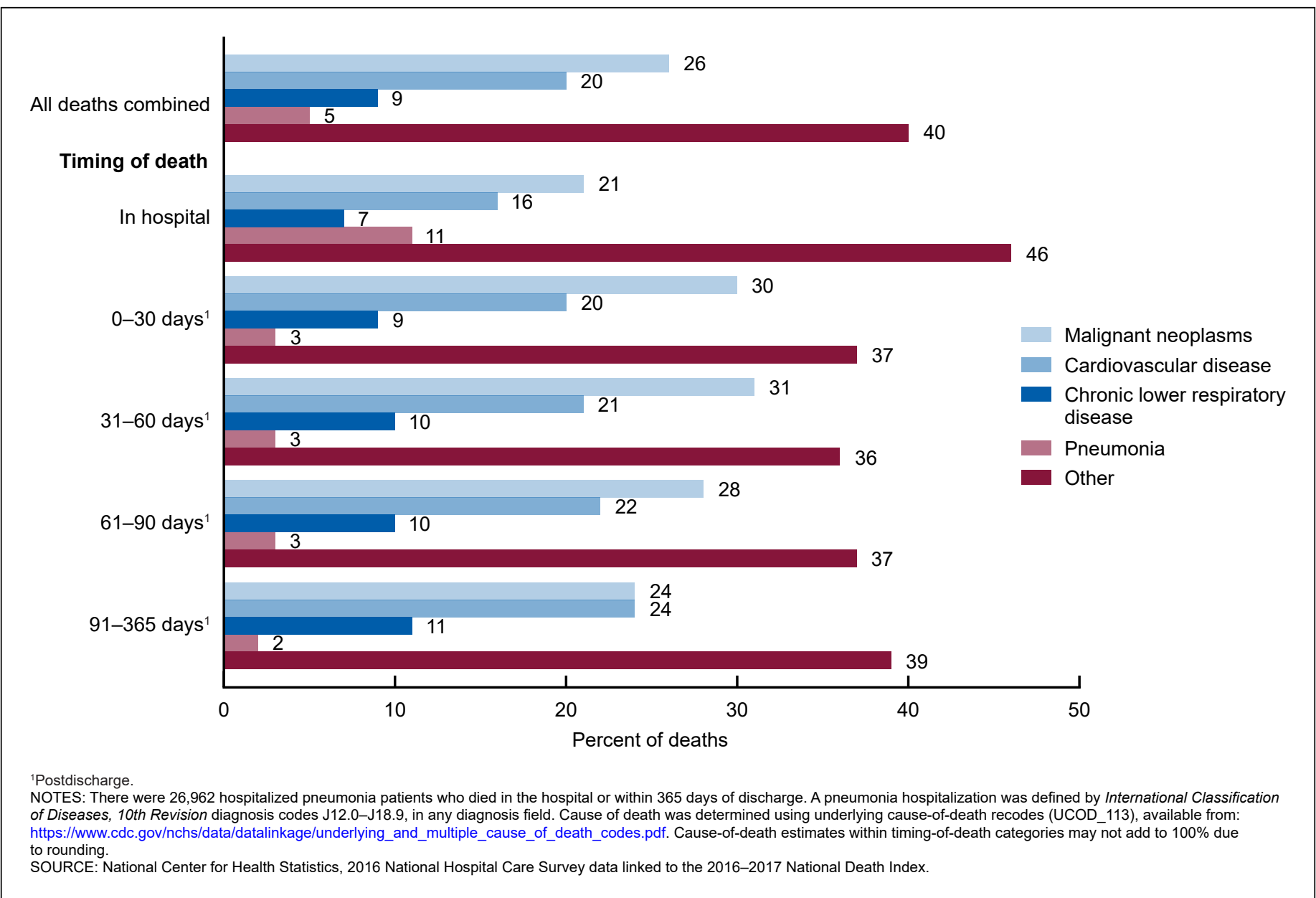

- Of pneumonia patients who were discharged alive from the hospital, those who were admitted to ICU while hospitalized had a $30 \%$ increased risk of death within 1 year than patients who were not admitted to ICU (RR: $1.30,95 \%$ CI: 1.27-1.33).

Table 4 depicts adjusted risk of death within 30 days of discharge among pneumonia patients who were discharged from the hospital, modeled by sex, age, length of stay, and ICU status.

- Male patients had a $21 \%$ higher risk of death within 30 days than female patients (adjusted RR [aRR]: 1.21, 95\% CI: 1.16-1.26), after adjusting for age, length of stay, and ICU status.

- For every 1-year increase in age, risk of death within 30 days of discharge among pneumonia patients increased by $4 \%$ after adjusting for sex, length of stay, and ICU status (aRR: 1.04, 95\% CI: 1.04-1.04).
- For every 1-day increase in length of stay, risk of death within 30 days of discharge among pneumonia patients increased by $2 \%$ after adjusting for sex, age, and ICU status (aRR: 1.02, 95\% CI: 1.02-1.03).

- Patients who were admitted to ICU while hospitalized for pneumonia had a $41 \%$ higher risk of death within 30 days than patients who were not admitted to ICU (aRR: $1.41,95 \%$ CI: $1.35-1.48)$, after adjusting for sex, age, and length of stay.

\section{Summary}

This report adds to previous efforts (19-22) that describe and demonstrate the capability of NHCS data linked to NDI to assess relationships between hospital care experiences and subsequent mortality. Although descriptive in nature, these analyses showcase the richness of these linked data in describing in-hospital and post-acute mortality for specific patient populations, as well as assessing mortality risk based on patient and hospitalization characteristics.

In this report, the results showed that over one-third of patients hospitalized for pneumonia in the 2016 NHCS died in the hospital or within 1 year of discharge, which is somewhat higher than other cohort studies from this timeframe that measured all-cause mortality among patients hospitalized for pneumonia 1 year postdischarge $(5,11)$. However, these studies were also not nationally representative and had stricter inclusion criteria for their cohort, such as requiring a primary pneumonia diagnosis or radiological evidence of pneumonia. Additionally, one study did not use linked mortality data (11) and the other assessed pneumonia hospitalizations from only one city in the United States (5), all of which likely contributed to differences in mortality rates.

Results in this report also showed that only $5 \%$ of deaths among patients 
hospitalized for pneumonia listed pneumonia as an underlying or contributing cause of death. Studies have also found pneumonia to be an infrequent contributing cause of death for pneumonia patients who died in the hospital (23) or postdischarge (24). Lastly, these linked data allow for the assessment of risk of death using both patient and hospitalization characteristics. For example, pneumonia patients who were admitted to ICU were four times more likely to die in the hospital than patients not admitted to ICU; even among patients who were discharged alive, admission to ICU predicted risk of death within 1 year. This aligns with previous studies that have shown increased risk of mortality among pneumonia patients admitted to $\operatorname{ICU}(9,25)$ or as length of ICU stay increased (8).

Findings should be considered within the context of this study's limitations. First, the 2016 NHCS data are not nationally representative and are therefore not generalizable beyond the responding sample of hospitals. Second, models of mortality risk included a small number of predictor variables; other contributing factors that were not or could not be assessed in this study may further explain these results. Additionally, not all NHCS sampled hospitals provided data on primary or first-listed diagnoses or whether diagnoses were present on admission. Consequently, this study could neither assess hospitalizations with pneumonia as a primary diagnosis nor distinguish between hospitalizations for community-acquired compared with hospital-acquired pneumonia. Lastly, this study lacked the ability to examine findings by patient race and ethnicity, as the 2016 NHCS did not collect this information.

NCHS expects the number hospitals participating in NHCS to increase in future years, as it moves toward collecting patient data through electronic health records. As participation in NHCS grows and these data become more representative, data linked to NDI will provide more comprehensive assessment of the relationships between hospital care utilization and mortality among various patient cohorts.

\section{About the authors}

Zachary J. Peters, Jill J. Ashman, Alexander Schwartzman, and Carol J. DeFrances are with the National Center for Health Statistics, Division of Health Care Statistics.

\section{References}

1. Hines AL, Heslin KC, Jiang HJ, Coffey R. Trends in observed adult inpatient mortality for high-volume conditions, 2002-2012. Healthcare Cost and Utilization Project Statistical Brief, no 194. 2015.

2. Hall MJ, Levant S, DeFrances C. Trends in inpatient hospital deaths: National Hospital Discharge Survey, 2000-2010. NCHS Data Brief, no 118. Hyattsville, MD: National Center for Health Statistics. 2013.

3. Agency for Healthcare Research and Quality. Healthcare cost and utilization project fast stats-Most common diagnoses for inpatient stays. 2021. Available from: https:/www. hcup-us.ahrq.gov/faststats/NationalD iagnosesServlet?year $1=2018 \&$ charac teristic $1=0 \&$ included $1=0 \&$ year $2=\& \mathrm{c}$ haracteristic $2=0 \&$ included $2=1 \&$ expa nsionInfoState $=$ hide $\&$ dataTablesState $=$ hide\&definitions State $=$ hide\&export State $=$ hide.

4. Xu JQ, Murphy SL, Kochanek KD, Arias E. Mortality in the United States, 2018. NCHS Data Brief, no 355. Hyattsville, MD: National Center for Health Statistics. 2020.

5. Ramirez JA, Wiemken TL, Peyrani P, Arnold FW, Kelley R, Mattingly WA, et al. Adults hospitalized with pneumonia in the United States: Incidence, epidemiology, and mortality. Clin Infect Dis 65(11):1806-12. 2017.

6. Ruhnke GW, Coca-Perraillon M, Kitch BT, Cutler DM. Marked reduction in 30-day mortality among elderly patients with communityacquired pneumonia. Am J Med 124(2):171-8.e1. 2011.

7. Falcone M, Tiseo G, Russo A, Giordo L, Manzini E, Bertazzoni G, et al. Hospitalization for pneumonia is associated with decreased 1-year survival in patients with Type 2 Diabetes: Results from a prospective cohort study. Medicine 95(5):e2531. 2016.

8. Moitra VK, Guerra C, Linde-Zwirble WT, Wunsch H. Relationship between ICU length of stay and long-term mortality for elderly ICU survivors. Crit Care Med 44:655-62. 2016.

9. Restrepo MI, Mortensen EM, Rello J, Brody J, Anzueto A. Late admission to the ICU in patients with community-acquired pneumonia is associated with higher mortality. Chest 137(3):552-7. 2010.

10. Peyrani P, Arnold FW, Bordon J, Furmanek S, Luna CM, Cavallazzi R, Ramirez J. Incidence and mortality of adults hospitalized with communityacquired pneumonia according to clinical course. Chest 157:34-41. 2020.

11. Averin A, Shaff M, Weycker D, Lonshteyn A, Sato R, Pelton SI. Mortality and readmission in the year following hospitalization for pneumonia among US adults. Respir Med 185:106476. 2021.

12. National Center for Health Statistics. National Hospital Care Survey. Available from: https://www.cdc.gov/ nchs/nhcs/about_nhcs.htm.

13. National Center for Health Statistics. National Death Index. Available from: https://www.cdc.gov/nchs/ndi/index. htm.

14. Fellegi IP, Sunter AB. A theory for record linkage. J Am Stat Assoc 64(328):1183-210. 1969.

15. National Center for Health Statistics. Office of Analysis and Epidemiology. The linkage of the 2016 National Hospital Care Survey to the 2016/2017 National Death Index: Methodology overview and analytic considerations, January 2019. Hyattsville, Maryland. Available from: https://www.cdc.gov/nchs/data/ datalinkage/NHCS16_NDI16_17_ Methodology_Analytic_Consider.pdf.

16. National Center for Health Statistics. Underlying and multiple cause of death codes. 2018. Available from: https://www.cdc.gov/nchs/ data/datalinkage/underlying_and multiple_cause_of_death_codes.pdf.

17.Zou G. A modified Poisson regression approach to prospective studies with binary data. Am J Epidemiol 159(7): 702-6. 2004. 
18. Knol MJ, Le Cessie S, Algra A, Vandenbroucke JP, Groenwold RHH. Overestimation of risk ratios by odds ratios in trials and cohort studies: Alternatives to logistic regression. CMAJ 184(8):895-9. 2012.

19. Spencer MR, Flagg LA, Jackson G, DeFrances C, Hedegaard H. National Hospital Care Survey Demonstration Projects: Opioid-involved emergency department visits, hospitalizations, and deaths. National Health Statistics Reports; no 141. Hyattsville, MD: National Center for Health Statistics. 2020.

20. National Center for Health Statistics Research Data Center. Linked data on hospitalizations, mortality, and drugs: Data from the National Hospital Care Survey 2016, National Death Index 2016-2017, and the Drug-Involved Mortality 2016-2017. 2020. Available from: https://www.cdc.gov/nchs/data/ nhcs/Task-3-Doc-508.pdf.

21. Jackson G, Brown AM, DeFrances C. Opioid-involved emergency department visits in the National Hospital Care Survey and the National Hospital Ambulatory Medical Care Survey. National Health Statistics Reports; no 149. Hyattsville, MD: National Center for Health Statistics. 2020.

22. Ashman JJ, DeFrances CJ, Cairns C, Schwartzman A. Respiratory illness emergency department visits in the National Hospital Care Survey and the National Hospital Ambulatory Medical Care Survey. National Health Statistics Reports; no 151. Hyattsville, MD: National Center for Health Statistics. 2021.

23. Stefan MS, Jaber R, Lindenauer PK, Garb JL, Fitzgerald J, Rothberg MB. Death among patients hospitalized with pneumonia: Implications for hospital outcome measures. JAMA Intern Med 175(5):851-3. 2015.

24. Bruns AHW, Oosterheert JJ,

Cucciolillo MC, El Moussaoui R, Groenwold RHH, Prins JM, Hoepelman AIM. Cause-specific long-term mortality rates in patients recovered from community-acquired pneumonia as compared with the general Dutch population. Clin Microbiol Infect 17(5):763-8. 2011.
25. Mortensen EM, Restrepo MI, Anzueto A, Pugh JA. Antibiotic therapy and 48-hour mortality for patients with pneumonia. Am J Med 119(10):859-64. 2006. 
Table 1. Criteria for defining qualifying pneumonia hospitalizations and linkage-eligible patients

\begin{tabular}{|c|c|c|}
\hline Characteristic & $N$ & Percent \\
\hline \multicolumn{3}{|l|}{ Hospitalizations, total and by compounding inclusion criteria } \\
\hline Total hospitalizations in 2016 National Hospital Care Survey . . . . . . . . . & $2,591,722$ & 100 \\
\hline With a pneumonia diagnosis. . . . . . . . . . . . . . . . . & 159,945 & 6 \\
\hline Among patients aged 18 and over $\ldots \ldots \ldots \ldots \ldots \ldots \ldots$ & 144,317 & 6 \\
\hline With complete sex and discharge data (qualifying hospitalizations) . . . & 130,657 & 5 \\
\hline Among patients eligible to be linked to the National Death Index $\ldots \ldots \ldots \ldots \ldots \ldots$ & 89,448 & 4 \\
\hline \multicolumn{3}{|l|}{ Patients, total and by mortality status } \\
\hline Total linkage-eligible patients with a qualifying pneumonia hospitalization. . . . . . . . . . & 76,857 & 100 \\
\hline Who died in hospital or within 1 year of discharge $\ldots \ldots \ldots \ldots \ldots \ldots \ldots$ & 26,962 & 35 \\
\hline 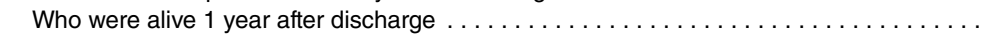 & 49,895 & 65 \\
\hline
\end{tabular}

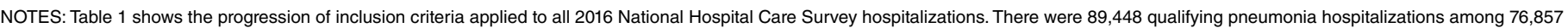
linkage-eligible patients. For patients who had more than one qualifying hospitalization, their most recent qualifying hospitalization was used for analysis.

SOURCE: National Center for Health Statistics, 2016 National Hospital Care Survey data linked to the 2016-2017 National Death Index.

Table 2. Risk of in-hospital death, by Intensive Care Unit status among all pneumonia patients

\begin{tabular}{|c|c|c|c|c|c|c|}
\hline \multirow[b]{2}{*}{ ICU admission } & \multicolumn{2}{|c|}{ In-hospital death } & \multirow[b]{2}{*}{ Risk } & \multirow[b]{2}{*}{ Risk ratio } & \multirow[b]{2}{*}{ Standard error } & \multirow{2}{*}{$\begin{array}{c}95 \% \text { confidence } \\
\text { interval }\end{array}$} \\
\hline & Yes & No & & & & \\
\hline Yes . . . . . . . . . . & 5,649 & 31,315 & 0.15 & 3.77 & 0.10 & $3.57-3.98$ \\
\hline No................ & 1,617 & 38,276 & 0.04 & $\ldots$ & $\ldots$ & $\ldots$ \\
\hline
\end{tabular}

... Category not applicable.

NOTES: ICU is intensive care unit. There were 76,857 linkage-eligible patients with a qualifying pneumonia hospitalization. Pneumonia was defined by International Classification of Diseases, 10 th Revision diagnosis codes between J12.0-J18.9, in any diagnosis field.

SOURCE: National Center for Health Statistics, 2016 National Hospital Care Survey data linked to the 2016-2017 National Death Index.

Table 3. Risk of death within 365 days, by Intensive Care Unit status among discharged pneumonia patients

\begin{tabular}{|c|c|c|c|c|c|c|}
\hline \multirow[b]{2}{*}{ ICU admission } & \multicolumn{2}{|c|}{ Post-acute death } & \multirow[b]{2}{*}{ Risk } & \multirow[b]{2}{*}{ Risk ratio } & \multirow[b]{2}{*}{ Standard error } & \multirow{2}{*}{$\begin{array}{c}95 \% \text { confidence } \\
\text { interval }\end{array}$} \\
\hline & Yes & No & & & & \\
\hline$\ldots \ldots \ldots \ldots \ldots$ & 10,150 & 21,165 & 0.32 & 1.30 & 0.02 & $1.27-1.33$ \\
\hline No............ & 9,546 & 28,730 & 0.25 & $\ldots$ & $\ldots$ & $\ldots$ \\
\hline
\end{tabular}

... Category not applicable.

NOTES: ICU is intensive care unit. There were 69,591 linkage-eligible patients with a qualifying pneumonia hospitalization who were discharged alive. Pneumonia was defined by International Classification of Diseases, 10th Revision diagnosis codes between J12.0-J18.9, in any diagnosis field.

SOURCE: National Center for Health Statistics, 2016 National Hospital Care Survey data linked to the 2016-2017 National Death Index.

Table 4. Risk of death within 30 days among discharged pneumonia patients

\begin{tabular}{|c|c|c|c|}
\hline Predictor & Adjusted risk ratio & Standard error & $\begin{array}{l}95 \% \text { confidence } \\
\text { interval }\end{array}$ \\
\hline Sex (male) $\ldots \ldots \ldots \ldots \ldots \ldots$ & 1.21 & 0.03 & $(1.16-1.26)$ \\
\hline Age $\ddagger \ldots \ldots \ldots \ldots \ldots \ldots \ldots$ & 1.04 & 0.00 & $(1.04-1.04)$ \\
\hline Length of stayt§ . . . . . . . . . . & 1.02 & 0.00 & $(1.02-1.03)$ \\
\hline ICU admission (yes) . . . . . . . . . . & 1.41 & 0.05 & $(1.35-1.48)$ \\
\hline Intercept $\ldots \ldots \ldots \ldots \ldots \ldots \ldots$ & -5.34 & 0.07 & $\ldots$ \\
\hline
\end{tabular}

$\dagger$ Continuous predictor variable.

† Patients over age 97 were set equal to 97 years (553 patients, $1 \%$ ).

$\S$ Hospitalization stays longer than 60 days were set equal to 60 days (480 patients, $1 \%$ ).

... Category not applicable.

NOTES: ICU is intensive care unit. There were 69,591 linkage-eligible patients with a qualifying pneumonia hospitalization who were discharged alive. Pneumonia was defined by International Classification of Diseases, 10th Revision diagnosis codes J12.0$\mathrm{J} 18.9$, in any diagnosis field. Risk ratios for individual predictors are adjusted for all other variables in this table.

SOURCE: National Center for Health Statistics, 2016 National Hospital Care Survey data linked to the 2016-2017 National Death Index. 


\section{U.S. DEPARTMENT OF \\ HEALTH \& HUMAN SERVICES}

FIRST CLASS MAIL

POSTAGE \& FEES PAID

CDC/NCHS

Centers for Disease Control and Prevention

PERMIT NO. G-284

National Center for Health Statistics

3311 Toledo Road, Room 4551, MS P08

Hyattsville, MD 20782-2064

OFFICIAL BUSINESS

PENALTY FOR PRIVATE USE, $\$ 300$

For more NCHS NHSRs, visit:

https://www.cdc.gov/nchs/products/nhsr.htm.

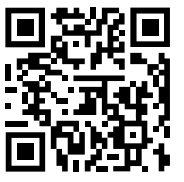

\section{Acknowledgments}

The authors thank Christine Cox, Linda Hermer, and Shawn Linman for their assistance in the early phase of this project.

\section{Suggested citation}

Peters ZJ, Ashman JJ, Schwartzman A, DeFrances CJ. National Hospital Care Survey demonstration projects: Examination of inpatient hospitalization and risk of mortality among patients diagnosed with pneumonia. National Health Statistics Reports; no 167. Hyattsville, MD: National Center for Health Statistics. 2022.

DOI: https://dx.doi.org/10.15620/cdc:112080.

\section{Copyright information}

All material appearing in this report is in the public domain and may be reproduced or copied without permission; citation as to source, however, is appreciated.

\section{National Center for Health Statistics}

Brian C. Moyer, Ph.D., Director

Amy M. Branum, Ph.D., Associate Director for Science

Division of Health Care Statistics

Carol J. DeFrances, Ph.D., Acting Director Alexander Strashny, Ph.D., Associate Director for Science

For e-mail updates on NCHS publication releases, subscribe online at: https://www.cdc.gov/nchs/email-updates.htm. For questions or general information about NCHS: Tel: 1-800-CDC-INFO (1-800-232-4636) • TTY: 1-888-232-6348 Internet: https://www.cdc.gov/nchs • Online request form: https://www.cdc.gov/info • CS328331 